\title{
On burning regimes and long duration X-ray bursts
}

\author{
L. Keek* \\ SRON Netherlands Institute for Space Research, Sorbonnelaan 2, NL - 3584 CA Utrecht, the \\ Netherlands \\ Astronomical Institute, Utrecht University, P.O. Box 80000, NL - 3508 TA Utrecht, the \\ Netherlands \\ E-mail: 1.keek@sron.nl

\section{J. J.M. in 't Zand} \\ SRON Netherlands Institute for Space Research, Sorbonnelaan 2, NL - 3584 CA Utrecht, the \\ Netherlands
}

\begin{abstract}
Hydrogen and helium accreted onto a neutron star undergo thermonuclear burning. Explosive burning is observed as a type I X-ray burst. We describe the different burning regimes and focus on some of the current inconsistencies between theory and observations. Of special interest are the rare kinds of X-ray bursts such as carbon-fueled superbursts and helium-fueled intermediately long X-ray bursts. These bursts are thought to originate deeper in the neutron star envelope, such that they are probes of the thermal properties of the crust. We investigate the possibility of observing superbursts with the wide-field instruments INTEGRAL-ISGRI and Swift-BAT. We find that only the brightest bursts are detectable.
\end{abstract}

7th INTEGRAL Workshop

September 8-112008

Copenhagen, Denmark

\footnotetext{
${ }^{*}$ Speaker.
} 


\section{Introduction}

Neutron stars in low-mass X-ray binaries can accrete material containing hydrogen and helium from a lower-mass companion star. Due to the high surface gravity of neutron stars, the material is compressed within a matter of hours to such high densities that, when it is just a few meters thick, the conditions for fusion of hydrogen and of helium are reached. If the thermonuclear fusion is unstable, the entire surface is burned within one second. Afterwards, the neutron star envelope cools down on a typical timescale of $10 \mathrm{~s}$. This is observable as a type I X-ray burst (Woosley \& Taam 1976; Maraschi \& Cavaliere 1977; Lamb \& Lamb 1978). The spectrum is usually well-fit by a simple black body with a peak temperature of 2 to $3 \mathrm{keV}$, which makes them detectable in the classical X-ray band. During the tail of the burst, the black body temperature decreases. Since their discovery (Grindlay et al. 1976), several thousands of these bursts have been observed from approximately 90 sources (e.g., in 't Zand et al. 2004b; Galloway et al. 2006; in 't Zand et al. 2007; for reviews see Lewin et al. 1993; Strohmayer \& Bildsten 2006). In this proceedings paper we focus on aspects of the burning processes responsible for X-ray bursts and on rare kinds of long bursts that have been discovered in recent years.

\section{Stable and unstable burning regimes}

Several nuclear reaction chains are responsible for the burning on a hydrogen/helium accreting neutron star. For instance, hydrogen burns to helium via the CNO cycle and helium burns to carbon through the triple-alpha process. The reaction rates of these processes can be strongly dependent on the temperature in the envelope, such that a small increase in the temperature causes a large rise in the energy generation rate. If the cooling rate is too low, this again increases the temperature, leading to a thermonuclear runaway, which is observable as an X-ray burst. At higher temperatures the dependence weakens, such that above a certain temperature burning proceeds in a stable manner. For hydrogen burning the transition is at $T \simeq 8 \cdot 10^{7} \mathrm{~K}$, while for helium burning it is $T \simeq 3.5 \cdot 10^{8} \mathrm{~K}$ (Bildsten 1998). Above this threshold, hydrogen burning follows a slightly different reaction chain known as the hot $\mathrm{CNO}$ cycle, which is temperature independent.

The temperature in the envelope is influenced by heating as well as cooling processes and increases with depth. The most important source of heat is stable thermonuclear burning of hydrogen and helium in the envelope itself. Cooling is mostly radiative. Part of the heat may be conducted inward, but in the envelope this is estimated to be only a small fraction (Eichler \& Cheng 1989). Another source of heat is the crust, a solid layer located below the envelope. During accretion the crust is compressed, which induces pycnonuclear ${ }^{1}$ reactions and electron captures. About $10 \%$ of the thus generated energy heats the envelope, while the rest is conducted deeper into the neutron star. The heat flux generated per accreted nucleon is thought to be $Q=0.15 \mathrm{MeV}$ nucleon $^{-1}$ (Haensel \& Zdunik 2003), although a recent study indicates it may be a few times higher (Gupta et al. 2007).

\footnotetext{
${ }^{1}$ In pycnonuclear reactions (first suggested by Gamow 1939; see, e.g., Yakovlev et al. 2005) the Coulomb barrier is penetrated due to zero-point vibrations of nuclei arranged in for instance a lattice, while in thermonuclear reactions, such as occur in X-ray bursts, it is penetrated by the thermal energy of colliding nuclei. Pycnonuclear reactions occur at high densities such as in neutron star crusts while thermonuclear reactions take place at high temperatures.
} 
Table 1: Burning regimes as a function of mass accretion rate (Fujimoto et al. 1981; Bildsten 1998), for a hydrogen/helium accreting neutron star mass of $1.4 \mathrm{M}_{\odot}$, a radius of $10 \mathrm{~km}$, a hydrogen mass fraction $X=0.7$ and a $\mathrm{CNO}$ mass fraction $Z_{\mathrm{CNO}}=0.01$.

\begin{tabular}{lcl}
\hline Regime & $\dot{M} / \dot{M}_{\text {Edd }}$ & Burning \\
\hline I & & Mixed H/He flash (H ignites first) \\
II & $0.5 \%$ & \\
& $3 \%$ & He flash (stable H burning) \\
III & & Mixed H/He flash (He ignites first) \\
IV & $100 \%$ & \\
\hline
\end{tabular}

The amount of heating depends on the mass accretion rate $\dot{M}$. For a higher $\dot{M}$ the heating by processes in the crust is stronger, leading to a higher temperature at the base of the burning layer. This influences the stability of thermonuclear burning, such that several burning regimes can be distinguished as a function of $\dot{M}$. In Table 1 we indicate the mass accretion rate at the transition between the regimes as found in models (Fujimoto et al. 1981; Bildsten 1998). All regimes are observed, but the mass accretion rate at the transitions is only approximately in agreement with the theoretical picture. For example, Cornelisse et al. (2003) observe the transition from regime I to II at $\dot{M} \simeq 0.1 \dot{M}_{\text {Edd }}$ (note that Galloway et al. 2004 argue this is the transition from regime II to III). van Paradijs et al. (1988) find indications of an increasing amount of stable $\mathrm{H} / \mathrm{He}$ burning at $\dot{M} \gtrsim 0.1 \dot{M}_{\text {Edd }}$, suggesting that there is a smooth transition from regime III to IV starting at a lower accretion rate than predicted by theory. The theoretical picture changes somewhat if certain important effects are incorporated in the models. In their models that include the effect of sedimentation Peng et al. (2007) find at low accretion rates, $0.3 \% \lesssim \dot{M} / \dot{M}_{\text {Edd }} \lesssim 1 \%$, a new regime of pure hydrogen bursts, where helium sinks to larger depths (see also Cooper \& Narayan 2007; in 't Zand et al. 2007). Another important effect is turbulent mixing due to rotation (Fujimoto 1993). When matter is accreted from the disk onto the neutron star it likely has a higher angular velocity than the neutron star itself. The accreted angular momentum is shared with the rest of the star. This leads to differential rotation, which causes turbulence that can mix the chemical composition of neighboring layers. If mixing is important, hydrogen and helium are diffused to larger depths, where the temperature is higher. This shifts the boundaries of the predicted burning regimes (Table 1). Yoon et al. (2004) investigated the effect of rotationally induced mixing on the stability of burning in the case of helium accreting white dwarfs. They find that the effect is important, as it leads to increased stability of thermonuclear burning. Piro \& Bildsten (2007) study helium accretion on neutron stars and find that also in this case mixing increases stability. Their models include a rotationally induced magnetic field, which very efficiently transports angular momentum through the envelope. Recently Keek et al. 2009 studied stable burning of helium in the neutron star envelope using a one dimensional hydrodynamic stellar evolution code including the effect of both rotational mixing and a rotationally induced magnetic field. They find that stability may be increased even further than Piro \& Bildsten (2007) predict, as the stabilizing effect is substantial for models at all rotation rates. Combined with the increased heat flux from the crust predicted by Gupta et al. 
Table 2: Comparison of the typical properties of different kinds of type I bursts.

\begin{tabular}{llll}
\hline & normal & intermediate & superburst \\
\hline duration & $10-100$ seconds & $15-40$ minutes & 1 day \\
fluence & $10^{39} \mathrm{erg}$ & $10^{40}-10^{41} \mathrm{erg}$ & $10^{42} \mathrm{erg}$ \\
recurrence time & hours-days & tens of days & 1 year \\
number observed & $1000 \mathrm{~s}$ & $\sim 20$ & 15 \\
& from $\sim 90$ sources & from 8 sources & from 10 sources \\
\hline
\end{tabular}

(2007), these effects may enable us to better explain the observed burning behavior as a function of mass accretion rate.

\section{Burst duration}

From our overview of the burning regimes (Table 1), we see that flashes may occur in burning layers that have different relative hydrogen and helium contents. Observationally we can discriminate between these bursts using their duration. The duration of a burst is set by the cooling time scale of the burning layer, which is determined by the depth at which the burning takes place. Typically this is of the order of $10 \mathrm{~s}$. In the presence of hydrogen, however, prolonged nuclear burning can extend the burst duration up to around $100 \mathrm{~s}$. A series of proton captures and $\beta$-decays creates heavy elements with mass numbers of up to 100 (e.g., Schatz et al. 2003; Fisker et al. 2008). This is known as the rp-process. Therefore, bursts last longer when the fuel has a larger hydrogen content. In a histogram of the burst decay time for a large number of type I bursts (see in 't Zand 2007 or Chenevez et al. 2008 in these proceedings) we see a broad distribution of bursts with exponential decay times of a few seconds up to $100 \mathrm{~s}$. Surprisingly, there are also two groups of bursts with much longer decay times of up to $10^{4} \mathrm{~s}$ : the so-called intermediate bursts and the superbursts. Their decay times are too long to be explained by prolonged burning through the rp-process. Therefore, these represent the cooling time scales of very thick layers. The thicker layers imply higher ignition pressures and, thus, different ignition temperatures or fuel compositions. Indeed, intermediately long bursts are thought to be due to much thicker hydrogen/helium layers on colder neutron stars (in 't Zand et al. 2005; Cumming et al. 2006) and superbursts are thought to result from carbon burning (Cornelisse et al. 2000; Cumming \& Bildsten 2001; Strohmayer \& Brown 2002). Comparing the properties of these different kinds of bursts in Table 2, we see that they are indeed consistent with the burning of thicker layers of fuel in the longer bursts, as their fluence is also higher. Since it takes a longer time to accumulate a thicker layer, the intermediate and superbursts have longer recurrence times, leading to a much lower number of observed instances than for normal bursts. For example, only 15 superbursts have been observed from 10 sources (Table 3). Therefore, most long duration bursts were observed relatively recently, after the launch of the observatories BeppoSAX and RXTE, which collected unprecedented amounts of exposure time on many of the bursting sources in our Galaxy.

\section{Intermediate bursts}

Most intermediate bursts are observed from so-called ultra-compact X-ray binaries (UCXBs), 
which have an orbital period of less than 80 minutes. In such a small orbit only a companion star fits which has lost its hydrogen-rich envelope. The neutron star, therefore, likely does not accrete any hydrogen, but may accrete helium (in 't Zand et al. 2005). Without stable hydrogen burning, the envelope is relatively cold, such that a much thicker layer has to be accreted before the ignition conditions for helium burning are reached (Cumming et al. 2006). Furthermore, the most important source of heat is in this case crustal heating. This makes intermediate bursts probes of the neutron star crust while mass accretion is ongoing.

A thick helium layer could also form on a hydrogen-accreting neutron star, if at low mass accretion rates pure hydrogen bursts occur, while sedimentation causes helium to settle at larger depths (Peng et al. 2007). The hydrogen bursts would be too weak to ignite the helium layer. Furthermore, these bursts may not exceed the accretion luminosity and may therefore not be detectable. Two intermediate bursts have been argued to be due to this scenario: a burst from the globular cluster M28 (Gotthelf \& Kulkarni 1997; Peng et al. 2007) and one from IGR 17254-3257 (Chenevez et al. 2007). in 't Zand et al. 2007 point out some issues which may prevent pure hydrogen bursts from occurring.

Intermediately long bursts with durations of 6 to 25 minutes have also been observed from GX 17+2 (Kuulkers et al. 2002a). These are interesting cases, since the source is not known to be an UCXB and it accretes at near-Eddington rates, while most UCXBs accrete at relatively low rates. Furthermore, this source exhibits short $(\sim 10 \mathrm{~s})$ bursts as well, which are often attributed to the unstable burning of a relatively thin helium layer. The size of the layer is determined by the depth at which the ignition conditions for helium are reached. It is unclear why for some bursts a thin layer is sufficient, while for others a thick layer is required. It appears more likely that in the intermediate bursts of GX $17+2$ we are witnessing a different burning regime than in the other intermediately long bursts.

\section{Questions about superbursts}

Superbursts are thought to originate in a 100 meter thick carbon-rich layer containing the ashes of hydrogen and helium burning. Most superbursting sources accrete hydrogen-rich material at a rate of $\dot{M} \gtrsim 0.1 \dot{M}_{\text {Edd }}$ (e.g., Kuulkers 2004). The base of the carbon-rich layer is close to the crust, which means that crustal heating is important for reaching the ignition temperature for carbon burning. Therefore, like intermediate bursts, superbursts are probes of the neutron star crust.

For a superburst to occur, two main ingredients are required: sufficient carbon to fuel the burst and a high enough temperature to ignite the flash. Carbon is thought to be created mostly by stable helium burning, but it is destroyed by the rp-process in the presence of hydrogen during bursts. Since all known superbursters also exhibit normal bursts, this presents a problem: how can sufficient carbon survive the many (at least hundreds) normal X-ray bursts before a superburst? Keek et al. (2008) suggest that sedimentation may separate carbon from the lighter elements hydrogen and helium, but this should be investigated further. While normal X-ray bursts pose the problem of removing carbon, this same process aids in reaching the required ignition temperature. The heavy elements that are created by proton captures on carbon, decrease the thermal conductivity of the envelope. By reducing the efficiency of cooling, this allows for the envelope to heat up faster, leading to shorter superburst recurrence times of approximately one year (Cumming \& Bildsten 


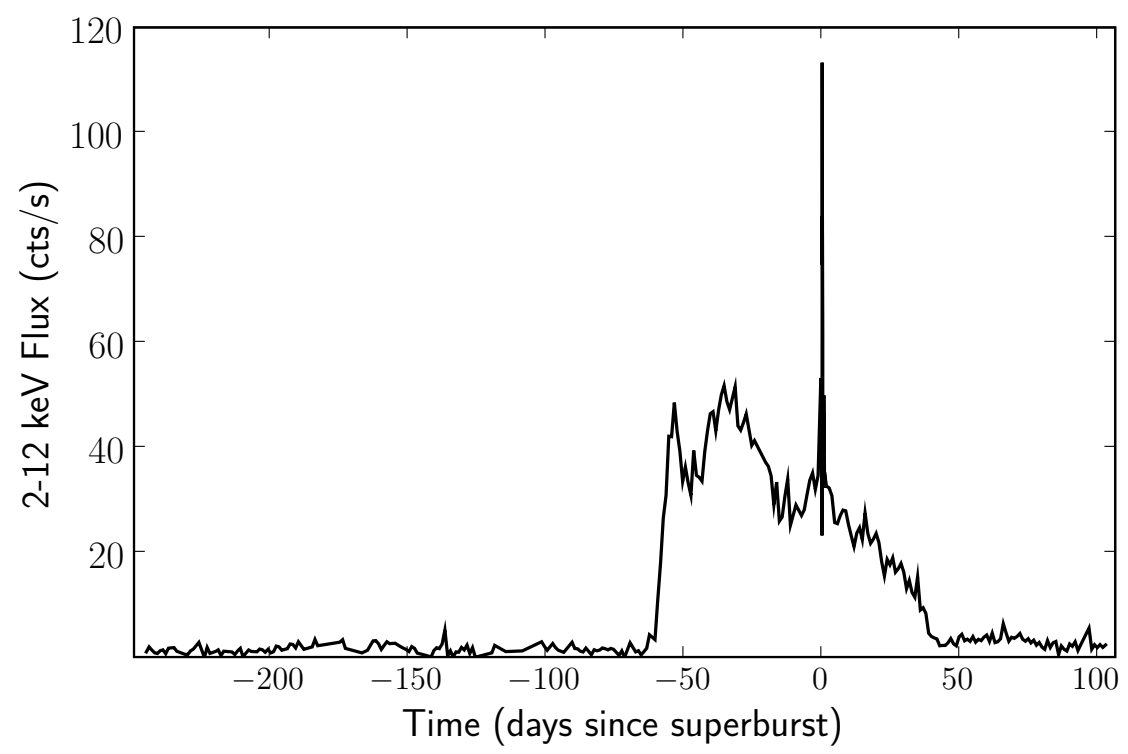

Figure 1: RXTE ASM light curve of 4U 1608-522 during the 2005 major outburst. 55 days after the start of the outburst a superburst occurred (Keek et al. 2008). Time resolution is two weeks, except during the superburst where the $90 \mathrm{~s}$ ASM dwells are used.

2001). This leads to the realization that for most superbursters both stable and unstable burning are required (in 't Zand et al. 2003; Schatz et al. 2003).

The problems in understanding how these two ingredients are obtained are well illustrated by the first observation of a superburst from the "classical'" transient 4U 1608-522 (Keek et al. 2008; Remillard et al. 2005). The neutron star started accreting at a high level only 55 days before the occurrence of the superburst (Fig. 1). This is not enough time to accumulate the required carbonrich layer, so the layer must have been formed during multiple outbursts in the previous 26 to 72 years. In the mean time, many normal type-I bursts occurred. Furthermore, during accretion the crust heats up, but according to the most recent models 55 days is not enough time to reach the ignition temperature for a carbon flash. Likely, there is an important extra source of heat in the crust, that is not taken into account yet.

\section{On the possibility of detecting superbursts with INTEGRAL-ISGRI and Swift-BAT}

While intermediate bursts have been detected with INTEGRAL-ISGRI and Swift-BAT (e.g., Chenevez et al. 2008 in these proceedings), no superbursts have been reported from observations with these instruments. In this section we investigate the possibility of detecting superbursts with IBIS/ISGRI and BAT.

\footnotetext{
${ }^{2}$ Previously, a superburst was observed from the transient KS 1731-260 (Kuulkers et al. 2002b). This source, however, accreted at a high rate for over ten years before the superburst was observed, in contrast to classical transients which accrete for only a fraction of a year.
} 
IBIS has a field of view of the same order as the BeppoSAX WFCs. The BeppoSAX instruments detected 4 superbursts in a typical exposure time of 6 to $12 \mathrm{Msec}$ per superburster (excluding the 4 superbursts from the luminous source GX 17+2, in 't Zand et al. 2004a). The IBIS exposure times are of the same order of magnitude. From this perspective one would expect a few superburst detections in the ISGRI data. However, the $>15 \mathrm{keV}$ bandpass of ISGRI is not ideal for the detection of few-keV black body spectra. For a black body temperature of 3.0/2.5/2.0/1.5 keV, the percentage of the photons above $15 \mathrm{keV}$ is $10 / 5 / 1.5 / 0.3 \%$ of the total. For a bolometric flux of $2 \times 10^{-8} \mathrm{erg} \mathrm{s}^{-1} \mathrm{~cm}^{-2}$, equivalent to roughly $1 \mathrm{Crab}$ between 2 and $10 \mathrm{keV}$, and a temperature of $\mathrm{k} T=2.5 \mathrm{keV}$, we expect an IBIS photon countrate of $15 \mathrm{c} / \mathrm{s}$ on axis. This number is based on a study of X-ray bursts in ISGRI data studied by Chelovekov et al. (2007) and on time-resolved spectroscopy on such data by Molkov et al. (2005) and Chenevez et al. (2007). If the same black body cools to $2.0 \mathrm{keV}$ (following the Stefan-Boltzmann law), this decreases to $2.5 \mathrm{c} / \mathrm{s}$. Note that due to its 3 to $35 \mathrm{keV}$ bandpass the JEM-X instrument on INTEGRAL is more favorable for observing superbursts. The smaller field of view, however, reduces the typical exposure time per superburster to $1 \mathrm{Msec}$, which implies that the average expected number of observed superbursts is less than one for this instrument.

We calculated ISGRI fluxes as a function of time for the superbursts seen from 4U 1820-303, $4 \mathrm{U}$ 1636-536, Ser X-1, 4U 1735-44, GX 3+1, 4U 1608-522 and 4U 0614+091, using the numbers in Table 3, and compared that to the 15-40 keV light curves as provided by the ISDC. It turns out that only 1 of the 7 superbursts could have been detected by ISGRI: that from 4U 1820-303. The significance with respect to the average error in the first superburst 'science window' (an exposure of 1800 to $3600 \mathrm{~s}$ ) would have been 40 and the flux 7 times higher than the average persistent flux. These high values are both due to the high peak temperature $(3 \mathrm{keV})$ and the large flux (2 times higher than the next brightest superburst). The other superbursts would have been fainter than the persistent flux, and have significances less than 4 per 'science window'.

We performed a similar study for the BAT on Swift, and obtain a similar conclusion. BAT is about twice less sensitive to X-ray bursts. Only the superburst from $4 \mathrm{U} 1820-303$ could have been detected, with a significance of about 20 in the first superburst orbit. Other superbursts have significances of 2 or less.

INTEGRAL has collected a large amount of exposure time on most (candidate) superbursters through a dedicated program to monitor the galactic center region (Kuulkers et al. 2007). Although Swift does not have a similar program, it still has collected a similar exposure time, since the field of view of BAT is eight times larger than that of IBIS. No superbursts are apparent from 4U 1820303 in 15.6 Msec of ISGRI (from February 28, 2003 up to November 11, 2006) nor $10.4 \mathrm{Msec}$ of BAT data (from February 12, 2005 up to November 11, 2008). Note that this is consistent with the longer recurrence time that is predicted for pure helium accretors (Strohmayer \& Brown 2002; see also Cumming 2003).

\section{Conclusion}

We reviewed the regimes of thermonuclear burning of accreted matter on neutron stars. Unstable burning of hydrogen and helium in the envelope is observable as type I X-ray bursts, which allow us to study the neutron star envelope. Since a few years we observe rare bursts with longer 
decay times of up to a few hours. The so-called intermediate bursts are due to thermonuclear burning of a thick helium layer, while the superbursts occur in a thick carbon-rich layer. The base of these thick layers lies close to the crust, such that the ignition of long bursts depends on the heating from the crust. Therefore, intermediate bursts and superbursts can be used as probes of the neutron star crust. The observation of one superburst - the first from a 'classical' transient — gives us important new insight in the thermal properties of the crust. Observing more long duration bursts will, therefore, allow us to improve and better constrain models of crustal heating. The wide-field instruments INTEGRAL-ISGRI and Swift-BAT have collected a large amount of exposure time on many of the bursting sources in our Galaxy. While these instruments have observed intermediate bursts, no superbursts were detected yet. We find that due to the energy range of the instruments, only very bright superbursts may be detected.

Acknowledgements. LK acknowledges support from The Netherlands Organization for Scientific Research (NWO). We thank Erik Kuulkers for providing us with details of the superburst from $4 \mathrm{U} 0614+91$ in advance of publication and for helpful comments. We are grateful to A. Parmar for careful refereeing of this paper and for interesting suggestions.

\section{References}

Bildsten, L. 1998, in NATO ASIC Proc. 515: The Many Faces of Neutron Stars., ed. R. Buccheri, J. van Paradijs, \& A. Alpar, 419

Chelovekov, I. V., Grebenev, S. A., \& Sunyaev, R. A. 2007, ArXiv e-prints, 709

Chenevez, J., Falanga, M., Kuulkers, E., et al. 2008, ArXiv e-prints

Chenevez, J., Falanga, M., Kuulkers, E., et al. 2007, A\&A, 469, L27

Cooper, R. L. \& Narayan, R. 2007, ApJ, 661, 468

Cornelisse, R., Heise, J., Kuulkers, E., Verbunt, F., \& in 't Zand, J. J. M. 2000, A\&A, 357, L21

Cornelisse, R., in 't Zand, J. J. M., Verbunt, F., et al. 2003, A\&A, 405, 1033

Cornelisse, R., Kuulkers, E., in’t Zand, J. J. M., Verbunt, F., \& Heise, J. 2002, A\&A, 382, 174

Cumming, A. 2003, ApJ, 595, 1077

Cumming, A. \& Bildsten, L. 2001, ApJ, 559, L127

Cumming, A., Macbeth, J., in 't Zand, J. J. M., \& Page, D. 2006, ApJ, 646, 429

Eichler, D. \& Cheng, A. F. 1989, ApJ, 336, 360

Fisker, J. L., Schatz, H., \& Thielemann, F.-K. 2008, ApJS, 174, 261

Fujimoto, M. Y. 1993, ApJ, 419, 768

Fujimoto, M. Y., Hanawa, T., \& Miyaji, S. 1981, ApJ, 247, 267 
Galloway, D. K., Cumming, A., Kuulkers, E., et al. 2004, ApJ, 601, 466

Galloway, D. K., Muno, M. P., Hartman, J. M., et al. 2006, ArXiv Astrophysics e-prints

Gamow, G. 1939, Physical Review, 55, 718

Gotthelf, E. V. \& Kulkarni, S. R. 1997, ApJ, 490, L161+

Grindlay, J., Gursky, H., Schnopper, H., et al. 1976, ApJ, 205, L127

Gupta, S., Brown, E. F., Schatz, H., Möller, P., \& Kratz, K.-L. 2007, ApJ, 662, 1188

Haensel, P. \& Zdunik, J. L. 2003, A\&A, 404, L33

in 't Zand, J. J. M. 2007, Observations of rare and peculiar X-ray bursts, presented at "The Neutron Star Crust and Surface: Observations and Models" in Seattle.

in 't Zand, J. J. M., Cornelisse, R., \& Cumming, A. 2004a, A\&A, 426, 257

in 't Zand, J. J. M., Cumming, A., van der Sluys, M. V., Verbunt, F., \& Pols, O. R. 2005, A\&A, 441, 675

in 't Zand, J. J. M., Jonker, P. G., \& Markwardt, C. B. 2007, A\&A, 465, 953

in 't Zand, J. J. M., Kuulkers, E., Verbunt, F., Heise, J., \& Cornelisse, R. 2003, A\&A, 411, L487

in 't Zand, J. J. M., Verbunt, F., Heise, J., et al. 2004b, Nucl. Phys. Proc. Suppl., 132, 486

Keek, L., in 't Zand, J. J. M., Kuulkers, E., et al. 2008, $A \& A$, 479, 177

Keek, L., Langer, N., \& in 't Zand, J. J. M. 2009, submitted for publication

Kuulkers, E. 2002, $A \& A, 383$, L5

Kuulkers, E. 2004, Nucl. Phys. Proc. Suppl., 132, 466

Kuulkers, E. 2005, The Astronomer's Telegram, 483, 1

Kuulkers, E., Homan, J., van der Klis, M., Lewin, W. H. G., \& Méndez, M. 2002a, A\&A, 382, 947

Kuulkers, E., in 't Zand, J., Homan, J., et al. 2004, in AIP Conf. Proc. 714: X-ray Timing 2003: Rossi and Beyond, 257-260

Kuulkers, E., in 't Zand, J. J. M., van Kerkwijk, M. H., et al. 2002b, A\&A, 382, 503

Kuulkers, E., Shaw, S. E., Paizis, A., et al. 2007, A\&A, 466, 595

Lamb, D. Q. \& Lamb, F. K. 1978, ApJ, 220, 291

Lewin, W. H. G., van Paradijs, J., \& Taam, R. E. 1993, Space Science Reviews, 62, 223

Maraschi, L. \& Cavaliere, A. 1977, in Highlights in Astronomy, ed. E. A. Müller, Vol. 4 (Reidel, Dordrecht), 127 
Molkov, S., Revnivtsev, M., Lutovinov, A., \& Sunyaev, R. 2005, A\&A, 434, 1069

Nelemans, G., Jonker, P. G., Marsh, T. R., \& van der Klis, M. 2004, MNRAS, 348, L7

Peng, F., Brown, E. F., \& Truran, J. W. 2007, ApJ, 654, 1022

Piro, A. L. \& Bildsten, L. 2007, ApJ, 663, 1252

Remillard, R., Morgan, E., \& The ASM Team at MIT, N. 2005, The Astronomer's Telegram, 482, 1

Schatz, H., Bildsten, L., Cumming, A., \& Ouelette, M. 2003, Nuclear Physics A, 718, 247

Strohmayer, T. \& Bildsten, L. 2006, New views of thermonuclear bursts (Compact stellar X-ray sources), 113-156

Strohmayer, T. E. \& Brown, E. F. 2002, ApJ, 566, 1045

Strohmayer, T. E. \& Markwardt, C. B. 2002, ApJ, 577, 337

van Paradijs, J., Penninx, W., \& Lewin, W. H. G. 1988, MNRAS, 233, 437

Wijnands, R. 2001, ApJ, 554, L59

Woosley, S. E. \& Taam, R. E. 1976, Nature, 263, 101

Yakovlev, D. G., Levenfish, K. P., \& Gnedin, O. Y. 2005, European Physical Journal A Supplement, 25,669

Yoon, S.-C., Langer, N., \& Scheithauer, S. 2004, A\&A, 425, 217 